them and their patients. All of us need to be continually reminded that some of the common therapeutic dilemmas can be resolved only by entering patients into controlled clinical trials.

Consultant Surgeon, Surgical Review Office,

I M C MACINTYRE

Western General Hospital, Edinburgh EH4 2XU

1 De Vita VT. Breast cancer therapy; exercising all our options. N Engl f Med 1989;320:527-9.

2 Jack WJL, Chetty U, Rodger A. Recruitment to a prospective conservation trial: why are so few patients randomised? BM7 1990;301:83-5.
3 Frieman JA, Thomas CC, Smith H, Kuebler RR. The importance of beta, the type II error and sample size in the design and interpretation of the randomized control trial. $N$ Engl $f$ Med sample size in

4 Medical Research Council. Streptomycin treatment of pulmonary tuberculosis. BMF 1948;ii:76982

5 Duncan AS, Dunstan GR, Welbourne RB, eds. Dictionary of medical ethics. London: Darton, Longman and Todd, 1981:132-5.

6 Taylor KM, Margolese RG, Soskolne CL. Physicians' reasons for not entering eligible patients in a randomised clinical trial of surgery for breast cancer. $N$ Engl F Med 1984;310:1363-7.

McKillop WJ, Palmer MJ, O'Sullivan B, Ward GK, Steele R, Dotsikos G. Clinical trials in cancer: the role of surrogate patients in defining what constitutes an ethically acceptable clinical experiment. Br $\mathcal{F}$ Cancer 1989;59:388-95.

8 Conn HO. Ideal treatment of portal hypertension in 1985. Clinical Gastroenterology 1985:14:259-88. 9 Karialainen S, Palva I. Do treatment protocols improve end results? A study of survival of patients with multiple myeloma in Finland. BMF 1989;299:1069-72.

\title{
Adjuvant therapy for cancer of the colon
}

\section{An important step forward}

Cancer of the colon and rectum is the second most common type of cancer in the Western world and is responsible for about 20000 deaths a year in Britain. The high death rate is frustrating for doctors - and tragic for patients. For though most patients are suitable for radical surgery, many relapse later because of microscopic disease undetectable at presentation. Some improvement has been seen in survival from cancer of the colon over the past few decades ( $R$ Doll, seventh King's Fund forum, 1990), but even so about half the patients who have "curative" resections will eventually die from their disease.

Might adjuvant chemotherapy improve survival in these circumstances? Its use after surgical resection in high risk groups has been shown to reduce mortality in several paediatric tumours. In breast cancer, adjuvant combination chemotherapy reduces mortality in premenopausal patients with positive nodes, while adjuvant tamoxifen has a similar effect in postmenopausal patients. ${ }^{12}$

Postoperative adjuvant therapy for colorectal cancer, usually with fluorouracil alone or in combination with other drugs, has been studied for more than 20 years. ${ }^{3}$ The treatments have been given for varying periods and using many different schedules, but the results have shown little benefit in individual trials. Recently, however, a metaanalysis which combined the data from all published trials suggested that regimens containing fluorouracil do, indeed, give a small survival benefit. ${ }^{3}$ The odds of death were $10 \%$ lower in treated than in control patients. When the analysis was restricted to trials in which chemotherapy was given over one year or more the gain was $17 \%$.

Recent studies from Britain and the United States have evaluated the combination of fluorouracil and levamisole. The first small study, in Glasgow, reported no difference in survival. ${ }^{+}$The second, from Leicester, randomised 141 patients to receive fluorouracil with or without postoperative levamisole or supportive care only. Deaths from colorectal cancer were significantly reduced in the patients receiving fluorouracil and levamisole. ${ }^{5}$ In 1989 the North Central Cancer Treatment Group and the Mayo Clinic published a study in which 401 patients with carcinoma of the colon (no patients with rectal cancer were included) were randomised either to a control arm or to treatment with levamisole alone or with levamisole plus fluorouracil. ${ }^{6}$ Both the patients receiving levamisole and fluorouracil and those treated with levamisole alone had a significantly reduced incidence of recurrence of their tumours compared with controls. There was also a survival advantage, which reached significance only in the subset of patients with stage $\mathrm{C}$ cancer of the colon treated with levamisole plus fluorouracil.
Encouraged by the early results from this study, the Eastern Cooperative Oncology Group, the North Central Treatment Group, the South West Oncology Group, and the Mayo Clinic initiated a larger trial (the intergroup trial). Nearly 1300 patients with stage B2 or C cancer of the colon were randomised among the same three treatment arms. With a median follow up of three years patients with stage $\mathrm{C}$ disease allocated fluorouracil and levamisole had a $41 \%$ reduction in the risk of recurrence and a reduction in mortality of $33 \%$. $^{7}$ This time levamisole alone had no detectable effect. Patients with stage $B 2$ disease had experienced too few events at the time of publication for firm conclusions to be drawn. The toxic effects of the combination of fluorouracil and levamisole were similar to those expected from fluorouracil alone-mild nausea and vomiting, mucositis, and diarrhoea. In addition a few patients developed myelosuppression and hair loss. A consensus meeting under the auspices of the National Cancer Institute held in Washington in May 1990 concluded that, though the optimal adjuvant therapy had not yet been devised, fluorouracil and levamisole as administered in the intergroup trial should be offered to all patients with stage $C$ cancer of the colon not entered into a clinical trial (unless they were medically or psychosocially unfit) ${ }^{8}$

The emphasis in these studies on the subgroup with stage $C$ disease may be criticised, but the trials were well conducted and the improvements in both the recurrence rate and survival in patients with stage $C$ disease were substantial. The studies did not, however, have patients treated with fluorouracil alone, though that drug might possibly have been the sole cause of the benefits shown. Though the recent metaanalysis of previous trials that used fluorouracil suggested a much smaller effect than the intergroup studies, the results are just statistically compatible with those of the intergroup studies. The intergroup studies used an intensive schedule of fluorouracil and documented a high level of compliance with the treatment, whereas previous trials used a variety of schedules and doses and there was no close control of compliance. The treatment actually received by the patients in the older studies might very possibly have been much less intensive, and this might account for the greater effect in the more recent studies. Whether levamisole plays any part in this combination clearly needs to be addressed in future trials.

The delivery of adjuvant chemotherapy into the intraportal route is another potentially fruitful avenue of research. In 1985 a randomised trial compared adjuvant chemotherapy with fluorouracil given for seven days into the portal vein with no treatment. The results showed a $60 \%$ reduction in the odds of death in favour of the treated group. ${ }^{9}$ Several later studies using similar regimens have shown smaller but still significant 
benefits. ${ }^{10-13}$ When the data from all published trials of intraportal treatment are combined they show an overall reduction in the odds of death of $26 \% .^{1+}$ Whereas some of the trials of intraportal fluorouracil showed a reduction in the incidence of liver metastases ${ }^{113}$ others showed no such reduction, ${ }^{1215}$ and the effect of this treatment might be systemic rather than a local effect on liver metastases. ${ }^{12}$ The perioperative approach to adjuvant chemotherapy does have the considerable advantage of a very short treatment period lasting only a few days - compared with one year of treatment in the intergroup studies. ${ }^{\top}$ The United Kingdom Coordinating Committee for Cancer Research is currently conducting AXIS, a new trial to attempt to confirm or refute the value of this approach, and this study will be crucial in determining the future of intraportal administration of fluorouracil.

Where do we go from here? The clear statement from the National Cancer Institute that adjuvant chemotherapy is now standard practice in stage $\mathrm{C}$ carcinoma of the colon and that "no treatment" controls are no longer justifiable has resulted in most doctors in the United States using fluorouracil and levamisole. ${ }^{1617}$ The attitude of European oncologists, however, is much more variable. ${ }^{1819}$ Though most are now more optimistic in their attitude to adjuvant therapy for cancer of the colon, many believe that studies have not yet established that the reduction in mortality with systemic chemotherapy outweighs the toxicity and inconvenience of the treatment and consider that trials should still include an untreated control arm. What is clear is that the pessimistic attitude to adjuvant therapy for cancer of the colon-common in the past - can no longer be justified. The recent intergroup trials from the United States were rigorous, and on the basis of these studies many clinicians in Britain are likely to conclude that those patients with stage $C$ cancer of the colon who are treated outside a clinical trial should be offered adjuvant therapy as used in the intergroup studies.

Much remains to be done-as the consensus report made clear. Newer treatments (such as the combination of fluorouracil with folinic acid, which has shown substantial activity in advanced cancer of the colon) should be tested as adjuvant therapy. The places of levamisole in combination with fluorouracil and of short perioperative infusions of fluorouracil into the portal vein need to be defined. Though it seems unlikely that the effect of adjuvant therapy in stage B cancer of the colon should be qualitatively different from that in stage $C$, the number of events and the follow up are not yet sufficient for any conclusions to be drawn from the published studies to date. Recent reports of adjuvant therapy in rectal cancer now suggest a degree of benefit similar to that in the colon cancer studies. ${ }^{20}$ The priority must now be to enter many more suitable patients with cancer of the colon into randomised trials of adjuvant therapy. Patients who are eligible for clinical trials should be encouraged to take part in such studies. Only by this means will progress be made. Individual doctors will have to judge whether or not they think a "no treatment" control arm is still justifiable, and patients will need to be informed of the issues before they are treated or offered randomisation.

Consultant Medical Oncologist,

MAURICE L SLEVIN

ICRF Department of Medical Oncology,

St Bartholomew's and Homerton Hospitals,

London EClA 7BE

Research Officer,

RICHARD GRAY

ICRF Cancer Studies Unit,

Radcliffe Infirmary,

Oxford OX2 6HE

1 Early Breast Cancer Trialists' Collaborative Group. Effects of adjuvant tamoxifen and of cytotoxic therapy on mortality in early breast cancer. An overview of 61 randomised trials among 28896 therapy on mortality in early breast cancer.
women. N Engl f Med 1988;319:1681-92.

2 Early Breast Cancer Trialists' Collaborative Group. Treatment of early breast cancer. Vol 1. Worldwide evidence 1985-1990. Oxford: Oxford University Press, 1990.

3 Buyse $M$, Zeleniuch-Jacquotte A, Chalmers TC. Adjuvant therapy of colorectal cancer. Why we still don't know. FAMA 1988;259:3571-8.

4 Bancewicz J, Calman K, MacPherson SG, McArdle C, McVie J, Soukop M. Adjuvant chemotherapy and immunotherapy of colorectal cancer. f $R$ Soc Med 1980;73:197-9.

Windle R, Bell PRF, Shaw D. Five year results of a randomised trial of adjuvant 5-fluorouracil and levamisole in colorectal cancer. Br f Surg 1987;74:569-72.

6 Laurie JA, Moertel CG, Fleming TR, el al. Surgical adjuvant therapy of large-bowel carcinoma: an evaluation of levamisole and the combination of levamisole and fluorouracil. $f$ Clin Oncology 1989;7:1447-56.

7 Moertel CG, Fleming TR, MacDonald JS, et al. Levamisole and fluorouracil for adjuvant therapy of resected colon carcinoma. $N$ Engl I Med 1990;322:352-8.

8 NIH Consensus Conference. Adjuvant therapy for patients with colon and rectal cancer. FAMA 1990;264:1444-50.

9 Taylor I, Machin D, Mullee M. Trotter (, , Cooke T, West C. A randomised control trial of adjuvant aylor I, Machin D, Mullee $M$, Trotter G, Cooke T, West C. A randomised control
portal vein cytotoxic perfusion in colorectal cancer. Br f S Surg 1985;72:359-63.

10 Gray BN, deZwart J, Fisher R, et al. The Australia and New Zealand trial of adjuvant therapy in colon cancer. In: Salmon SE, ed. Adjuzant therapy of cancer. Philadelphia: Grune and Stratton, 1987:357-456.

11 Metzger U, Mermillod B, Aeberhard P, et al. Intraportal chemotherapy in colorectal carcinoma as an adjuvant modality. World $\mathcal{F}$ Surg 1987;ii:452-8.

2 Wolmark N, Rockette H, Wickerham DL, ei al. Adjuvant therapy of Dukes' A, B and C adenocarcinoma of the colon with portal vein fluorouracil hepatic infusion: preliminary results of national surgical adjuvant breast and bowel project protocol C-02. F Clin Oncol 1990;8: 1466-75.

3 Wereldsma JCJ, Bruggink EDM, Meijer WS, et al. Adjuvant portal liver infusion in colorectal cancer with 5-fluorouracil/heparin versus urokinase versus control. Cancer 1990;64:425-32.

14 Gray R, James R, Mossman J. AXIS - a suitable case for treatment. UKCCCR Colorectal Cancer Sub-committee. Brf Cancer (in press).

15 Beart RW, Moertel CG, Wieand HS, et al. Adjuvant therapy for resectable colorectal carcinoma with fluorouracil administered by portal vein infusion. Arch Surg 1990;125:897-901.

16 Vanchieri C. Colon cancer update triggers changes in practice. F Natl Cancer Inst 1990;82:898.

16 Vanchieri C. Colon cancer update triggers changes in practice. F Natl Cancer Inst 1990;82:898 17 Mayer RJ. Does adjuvant therapy work in colon cancer? $N$ Engl $\mathcal{F}$ Med 1990;322:399-401
18 Anonymous. Mixed European reactions to American colorectal data. Annals of Oncologv

1990;1:239-40

9 Wills J, Wagener DJTH. Adjuvant treatment of colon cancer: where do we go from here? Annals of Oncology 1990;1:329-31.

20 Krook JE, Moertel CG, Gunderson L.L, et al. Effective surgical adjuvant therapy for high risk rectal carcinoma. $N$ Engl F Med 1991;324:709-15.

\section{How sick the baby?}

\section{A new method of assessment suggested}

One of the most worrying problems that general practitioners face is an acutely ill baby. Should they admit the baby to hospital? If the baby is not admitted will the parents call the doctor if the baby's condition deteriorates? A team from the department of paediatrics in Cambridge has now devised a method for assessing sick babies.

The Baby Check is intended to assess the severity of acute systemic illness in babies less than 6 months old. It consists of a checklist of 19 symptoms and signs, and the total score derived from these determines the recommended course of action. It is being promoted as a means whereby parents can decide when to call a doctor and for general practitioners to use to determine when specialist attention is needed. The Child Growth Foundation is currently distributing copies of Baby Check to some Family Health Service Authorities.

Will the Baby Check live up to its claims? How it was developed and tested is described in a series of papers in the Archives of Disease in Childhood. ${ }^{1-4}$ The symptoms and signs used to predict a poor outcome of illness were derived from one paediatrician's experience, but they accorded with those of other paediatricians. Because of the low incidence of severe illness in a community the instrument's sensitivity 